\title{
Characteristic properties of the nuclear magnetic resonance-paramagnetic relaxation enhancement arising from integer and half-integer electron spins
}

\author{
Robert R. Sharp \\ Department of Chemistry, The University of Michigan, Ann Arbor, Michigan, 48109
}

(Received 29 September 1992; accepted 5 November 1992)

\begin{abstract}
The influence of zero field splitting (zfs) interactions on the magnetic field dispersion profile of the nuclear magnetic resonance-paramagnetic relaxation (NMR-PRE) (i.e., the enhancement of nuclear magnetic relaxation rates that is produced by paramagnetic solute species in solution) has been explored systematically for $S=1,3 / 2,2$, and $5 / 2$ spin systems using recently developed theory. To facilitate comparison of results for different spin values, the theory was expressed in a reduced form with Larmor frequencies in units of $\omega_{D}$ (the uniaxial zfs parameter $D$ in $\mathrm{rad} \mathrm{s}^{-1}$ ), and correlation times and spin relaxation times in units of $\omega_{D}^{-1}$. For $S=1$, the functional form of the profile can be described in terms of five types of qualitative features. Two of these are characteristic of Zeeman-limit [Solomon, Bloembergen, and Morgan (SBM)] theory and result from the magnetic field dependence of the spin energy level splittings. The remaining three have no analog in Zeeman-limit theory and arise from a change in the quantization axis of the electron spin precessional motion which, in the zfs limit, lies along molecule-fixed coordinate axes, and, in the Zeeman limit, lies along the external field direction. The reduced field dispersion profiles for the integer spin systems $S=1$ and $S=2$ were found to be very similar to each other, the principal difference being that the midfield positions of the requantization features (types 2,3 , and 4 ) are shifted for $S=2$ relative to $S=1$, the magnitude and sign of the shift depending on the position of the nuclear spin in the molecular coordinate frame. For half-integer spins, the dispersion profiles exhibit, in addition to the five features characteristic of integer spins, a sixth type of feature, which is centered somewhat to low field of $\omega_{S} \tau_{c}=1$, where $\tau_{c}$ is the dipolar correlation time. The type- 6 feature results from field-dependent level splitting of the $m_{S}= \pm 1 / 2 \mathrm{Kram}$ ers doublet. It is present when $\omega_{D} \tau_{c}>1$. These theoretical predictions have been examined by means of reinterpretations of the NMR-PRE data for tris-(acetylacetonato)-metal complexes of V(III) $(S=1), \mathrm{Cr}$ (III) $(S=3 / 2), \mathrm{Mo}$ (III) $(S=3 / 2), \mathrm{Mn}$ (III) $(S=2)$, and $\mathrm{Fe}$ (III) ( $S$ $=5 / 2$ ). As predicted, type- 6 features are absent for the integer spin complexes, for which the $T_{1}$ field dispersion profiles are nearly field independent. The experimental profiles were successfully simulated quantitatively by the generalized theory, but not by Zeeman-limit theory. For the half-integer spin systems, the predicted zfs-related type- 6 features appear to be present in the profiles, particularly for $\mathrm{Mo}(a c a c)_{3}$, for which the data deviate significantly from the Zeeman-limit profile in a manner that is explained by the generalized theory.
\end{abstract}

\section{INTRODUCTION}

The nuclear spin resonances of paramagnetic molecules and complex ions in solution phase typically exhibit profound enhancements arising from magnetic interactions, both dipolar and scalar in origin, between the nuclear spins and unpaired electron spins. Large nuclear magnetic resonance-paramagnetic relaxation enhancements (NMR-PRE) also frequently appear in the nuclear spin resonances of the diamagnetic solvent and/or of diamagnetic solute species. These phenomena have been used widely over the past three decades in the study of chemical and magnetic properties of paramagnetic metal ions in chemical and biological systems. They are also of fundamental importance with respect to relaxation-related contrast mechanisms in NMR-imaging applications. ${ }^{1}$

The theory of paramagnetic relaxation enhancements derives from the seminal work of Solomon, ${ }^{2}$ Bloembergen, ${ }^{3,4}$ and Morgan. ${ }^{4}$ Their theory (SBM theory) is a lim- iting theory in that it assumes that the motion of the electron spin $S$ is a simple Zeeman precession, i.e., that the Zeeman term $\mathscr{H}_{Z}$ of the electron spin Hamiltonian $\mathscr{H}_{S}$ is large compared to the zero-field splitting Hamiltonian $\mathscr{H}_{\text {zfs }}$ plus the clcetron-nuclcar hyperfine Hamiltonian $\mathscr{H}_{\mathrm{hr}}$. The latter assumption $\mathscr{H}_{\mathrm{hf}} \ll \mathscr{H}_{Z}$ is normally very good, but the former $\mathscr{H}_{\mathrm{zfs}} \ll \mathscr{H}_{Z}$ is often poor or questionable. When the zfs interaction is large $\left(\mathscr{H}_{\mathrm{zfs}} \gg \mathscr{H}_{Z}\right)$, the quantization axes of the electronic precessional motion coincide with the molecule-fixed principal axis system of the zfs tensor rather than with the direction of the external magnetic field $B_{0}$, as in the Zeeman limit $\left(\mathscr{H}_{\text {zfs }} \ll \mathscr{H}_{Z}\right)$. The difference in quantization axes in the two limits profoundly influences NMR-PRE phenomenon, particularly with respect to magnetic field dependence of the NMR-PRE.

Previous papers in this series ${ }^{5-8}$ have explored systematically the effect of $\mathscr{H}_{\text {zfs }}$ on the NMR-PRE. Closed-form algebraic expressions which describe the intramolecular ${ }^{5}$ 
and intermolecular ${ }^{6}$ NMR-PRE in the zfs limit have been derived. These expressions are valid for arbitrary $S$ and parallel the form of the Zeeman-limit (SBM) theory. Subsequently, the theory was generalized to account for Zeeman and zfs interactions of arbitrary magnitude. ${ }^{7}$ The generalized theory is suitable for numerical evaluation and has been implemented in the program PARELAX. Calculations based on these theories have shown that, for $S=1$, zfs interactions have profound effects on the field dispersion profile. For $S=1$, the qualitative form of the dispersion profile can be described in terms of five distinct features, each with distinct functional characteristics. Two of the features are characteristic of the Zeeman limit; the other three types (types 2-4) arise from effects of the requantization of the electron spin. The properties of these features are described in Ref. 8. The present communication describes the structures of the field dispersion profiles for $S=3 / 2,2$, and $5 / 2$, comparing these with the structural features for $S=1$. It is shown that for integer spins $(S=1$ and $S=2$ ), the qualitative forms of the profiles are similar to each other and they can be described quite well in terms of the five types of features described previously. For halfinteger spins $(S=3 / 5$ and $S=5 / 2)$, the situation is somewhat more complex in that a new type of feature (type 6) occurs. This feature arises specifically from the $m_{S}= \pm 1 / 2$ Kramers doublet and is a characteristic feature of halfinteger spins systems in the vicinity of the zfs limit.

Earlier and rather different theoretical approaches to this problem are described in Refs. 9-20.

\section{METHODS}

The paramagnetic relaxation enhancement of a nuclear spin in the coordination complex of a paramagnetic metal ion results from time dependence in the dipolar and scalar parts of the electron-nuclear hyperfine energy $R_{1 m}$ $=R_{1 m, \text { dip }}+R_{1 m, \mathrm{sc}}$. From zfs-limit theory, ${ }^{5,6}$

$$
\begin{aligned}
R_{1 m, \mathrm{dip}}^{(\mathrm{zfs})}= & \frac{4}{3}\left(\frac{\gamma_{I} g \beta_{0}}{r^{3}}\right)^{2}\left(\frac{\mu_{0}}{4 \pi}\right)^{2}\left\{\frac{S(S+1)}{3}\left[1+P_{2}(\cos \Theta)\right]\right. \\
& \left.\times j\left(\omega_{I}\right)+\left[1-2^{-1} P_{2}(\cos \Theta)\right] \mathrm{J}_{S}\right\}
\end{aligned}
$$

where

$$
J_{S} \equiv(2 S+1)^{-1} \sum_{\mu}\left|c_{S, \mu}^{+}\right|^{2} j\left(\omega_{\mu}\right),
$$

$\gamma_{I}$ is the magnetogyric ratio of the $I$ spin, $\omega_{I}=\gamma_{I} B_{0}$ is the nuclear Larmor frequency, $g$ is the electronic $g$ factor, $\beta_{0}$ is the Bohr magneton, $\mu_{0}$ is the magnetic permeability of free space, $r$ is the $I-S$ distance, $\Theta$ is the polar angle of the $I-S$ vector in the molecular coordinate frame, and $P_{2}(\cos \theta)$ is the second order Legendre polynomial in $\cos \theta . j(\omega)=\tau_{c}$ d $\left(1+\omega^{2} \tau_{c}^{2}\right)$ is the spectral density function of the nuclearelectron magnetic dipole interaction, for which the correlation time is $\tau_{c}$. The sum in Eq. (2) is over the basis states $|\mu\rangle$ of $\mathscr{H}_{\mathrm{zfs}}$, and $c_{S, \mu}^{+}$is the raising operator of the $S$ spin manifold. The Zeeman-limit expression corresponding to Eq. (1) is ${ }^{2}$

$$
\begin{aligned}
R_{\mathrm{l} m, \mathrm{dip}}^{(Z)}= & \left(\frac{2}{15}\right)\left(\frac{\mu_{0}}{4 \pi}\right)^{2}\left(\frac{\gamma_{I} g \beta_{0}}{r^{3}}\right)^{2} S(S+1)\left[3 j_{1}\left(\omega_{I}\right)\right. \\
& \left.+j_{2}\left(\omega_{S}-\omega_{I}\right)+6 j_{2}\left(\omega_{S}+\omega_{I}\right)\right]
\end{aligned}
$$

where $\omega_{S}$ and $\omega_{I}$ are the electronic and nuclear Larmor frequencies.

Dipolar magnetic coupling of $I$ and $S$ spins produces relaxation transitions in the form of both single quantum transitions of the $I$ spin $\Delta m_{I}= \pm 1$ and in the form of coupled one quantum transitions of the $I$ and $S$ spins $\Delta m_{I}$ $= \pm 1$ and $\Delta m_{S}= \pm 1, \mp 1$. In Zeeman limit theory, these terms correspond, respectively, to the parts of Eq. (3) that are proportional to $j\left(\omega_{I}\right)$ and $j\left(\omega_{S} \pm \omega_{I}\right)$. In the zfs-limit expressions (1) and (2), they correspond to low frequency terms proportional to $j\left(\omega_{I}\right)$ and high frequency terms proportional to the spectral density function $j\left(\omega_{\mu}\right)$ at the one quantum zfs transition frequencies $\omega_{\mu}$. In the zfs limit, all the $\Delta m_{S}= \pm 1$ transition frequencies are integral multiples of $\omega_{D} \equiv 2 \pi c D$, the sole exception being the one quantum transition that joins the levels of the $m_{S}= \pm 1 / 2$ Kramers doublet in half-integer spin manifolds. The $m_{S}= \pm 1 / 2$ Kramers doublet is strictly degenerate in zero field, so that $\omega_{\mu}=\omega_{1 / 2,-1 / 2} \rightarrow 0$ as the field goes to zero.

Specific forms of the high frequency term $J_{S}$ in the zfs limit expression are tabulated here for $S=1$ through $S=5$ / 2:

$S=1$. Two transitions contribute with (1) $\mu=-1, \omega_{\mu}$ $=\omega_{D},\left|c_{1,-1}^{+}\right|^{2}=2$; and (2) $\mu=0, \omega_{\mu}=\omega_{D},\left|c_{1,0}^{1}\right|^{2}=2$ giving

$$
J_{1}=(4 / 3) j\left(\omega_{D}\right) .
$$

$S=3 / 2$. Three transitions contribute with (1) $\mu$ $=-3 / 2, \omega_{\mu}=2 \omega_{D}, \quad\left|c_{3 / 2,-3 / 2}^{+}\right|^{2}=3$; (2) $\mu=-1 / 2, \omega_{\mu}$ $=\omega_{1 / 2}, \quad\left|c_{3 / 2,-1 / 2}^{+}\right|^{2}=4 ;$ and (3) $\mu=+1 / 2, \omega_{\mu}=2 \omega_{D}$, $\left|c_{3 / 2,+1 / 2}^{+}\right|^{2}=3$ giving

$$
J_{3 / 2}=(3 / 2) j\left(2 \omega_{D}\right)+j\left(\omega_{1 / 2}\right) .
$$

$S=2$. Four electronic transitions contribute with (1) $\mu=-2, \quad \omega_{\mu}=3 \omega_{D}, \quad\left|c_{2,-2}^{+}\right|^{2}=4 ; \quad$ (2) $\mu=-1, \omega_{\mu}=\omega_{D}$, $\left|c_{2,-1}^{+}\right|^{2}=6$; (3) $\mu=0, \omega_{\mu}=\omega_{D}, \quad\left|c_{2,0}^{+}\right|^{2}=6$; and (4) $\mu=+1, \omega_{\mu}=3 \omega_{D},\left|c_{2,+1}^{+}\right|^{2}=4$ giving

$$
\mathrm{J}_{2}=(8 / 5) j\left(3 \omega_{D}\right)+(12 / 5) j\left(\omega_{D}\right) \text {. }
$$

$S=5 / 2$. Five electronic transitions contribute with (1) $\mu=-5 / 2, \omega_{\mu}=4 \omega_{D},\left|c_{5 / 2,-5 / 2}^{+}\right|^{2}=5 ;$ (2) $\mu=-3 / 2, \omega_{\mu}$ $=2 \omega_{D}, \quad\left|c_{5 / 2,-3 / 2}^{+}\right|^{2}=8 ; \quad$ (3) $\quad \mu=-1 / 2, \quad \omega_{\mu}=\omega_{1 / 2}$, $\left|c_{5 / 2,-1 / 2}^{+}\right|^{2}=9$; (4) $\mu=+1 / 2, \omega_{\mu}=2 \omega_{D},\left|c_{5 / 2,+1 / 2}^{+}\right|^{2}=8$; and $(5) \mu=+3 / 2, \omega_{\mu}=4 \omega_{D},\left|c_{5 / 2,+3 / 2}^{\prime}\right|^{2}=5$ giving

$$
\mathrm{J}_{5 / 2}=(5 / 3) j\left(4 \omega_{D}\right)+(8 / 3) j\left(2 \omega_{D}\right)+(3 / 2) j\left(\omega_{1 / 2}\right) .
$$

The definition of the dipolar correlation time $\tau_{c}$ differs in the zfs and Zeeman limits. In the former,

$$
\left(\tau_{c}\right)_{\mathrm{zfs}}^{-1}=\left(\tau_{S 2}\right)^{-1}+\left(\tau_{R}^{(1)}\right)^{-1}
$$

and in the latter,

$$
\left(\tau_{c 1,2}\right)_{Z}^{-1}=\left(\tau_{S 1,2}\right)^{-1}+\left(\tau_{R}^{(2)}\right)^{-1},
$$


where $\tau_{R}^{(1,2)}$ are reorientational correlation times for first and second rank molecule-fixed tensors. According to classical diffusion theory, ${ }^{21} \tau_{R}^{(1)}=3 \tau_{R}^{(2)} \cdot \tau_{S 1}$ and $\tau_{S 2}$ are the electron spin relaxation times parallel to, and in the plane transverse to the axis of the electronic precessional motion. In general, these quantities may differ from each other and may also change when the spin system passes from the zfs to the Zeeman limit. When $\tau_{S}$ is short, the electron spin is tightly coupled to the thermal lattice and its relaxation is likely to be described well by a single parameter ${ }^{8} \tau_{S}=\tau_{S 1}$ $=\tau_{S 2}$. This assumption was used in a previous work and is likewise used in the analyses here.

In the intermediate regime of field strengths $\left(\mathscr{H}_{\text {zfs }}\right.$ $\left.\approx \mathscr{H}_{Z}\right), R_{1 m}$ was calculated using the computer program PARELAX, which is based on the theory of Ref. 7. According to Eqs. (5) and (6), the appropriate definition of $\tau_{R}$ changes when the spin system passes from the zfs to the Zeeman limit. PARELAX employs a parametrized form for $\tau_{R}$,

$$
\left(\tau_{R}\right)^{-1}=\chi\left(\tau_{R}^{(2)}\right)^{-1}+(1-\chi)\left(\tau_{R}^{(1)}\right)^{-1}
$$

with

$$
\chi \equiv \sin ^{2}\left(\frac{\pi}{2} \cdot \frac{\omega_{S}}{\omega_{S}+S \omega_{D}}\right)
$$

which ensures a smooth transition between the limiting forms, centered about the field value for which $\omega_{S}=S \omega_{D}$. The quantity $S \omega_{D}$ provides a good approximation of the average single quantum zfs transition frequency (see below), and the field at which $\omega_{S}=S \omega_{D}$ was taken as the cross-over field, where $\mathscr{H}_{\text {zfs }} \approx \mathscr{H}_{Z}$. For the purpose of comparing results from spin systems with different $S$, a reduced form of the theory was used in which the transition frequencies and correlation times were expressed in units of $\omega_{D}$ and $\omega_{D}^{-1}$, respectively. With dimensionless frequencies and correlation times, the calculated relaxation times are likewise dimensionless, $\omega_{D} R_{1 m}$, with $R_{1 m}$ given in the limiting situations by Eqs. (2)-(5). In comparative calculations involving different spin manifolds, a "fully reduced" relaxation rate defined by

$$
R_{1 m}^{*} \equiv\left[g^{2} \beta_{0}^{2} \gamma_{I}^{2} S(S+1)\left(\mu_{0} / 4 \pi\right)^{2}\right]^{-1} \omega_{D} R_{1 m}
$$

was used. Theoretical results are plotted as reduced field dispersion profiles, plots of $R_{1 m}^{*}$ vs $\omega_{S} / \omega_{D}$, in which the intermediate regime between the Zeeman and zfs limits corresponds approximately to the field strength for which $\omega_{S} / S \omega_{D}=1$.

\section{A. The five types of qualitative feature in field dispersion profiles of $S=1$}

To facilitate discussion of the field dispersion profiles for $S>1$, it is convenient first to summarize the properties of the five qualitative features which have previously been described for $S=1$ (8).

Type 1. These are the characteristic dispersions of Zeeman-limit theory, which arise from the magnetic field dependence of the spectral density functions $j\left(\omega_{I}\right)=\tau_{c} /(1$ $\left.+\omega_{I}^{2} \tau_{c}^{2}\right)$ and $j\left(\omega_{S} \pm \omega_{I}\right)=\tau_{c} /\left(1+\left[\omega_{S} \pm \omega_{I}\right]^{2} \tau_{c}^{2}\right)$. Low frequency and high frequency type- 1 features are centered

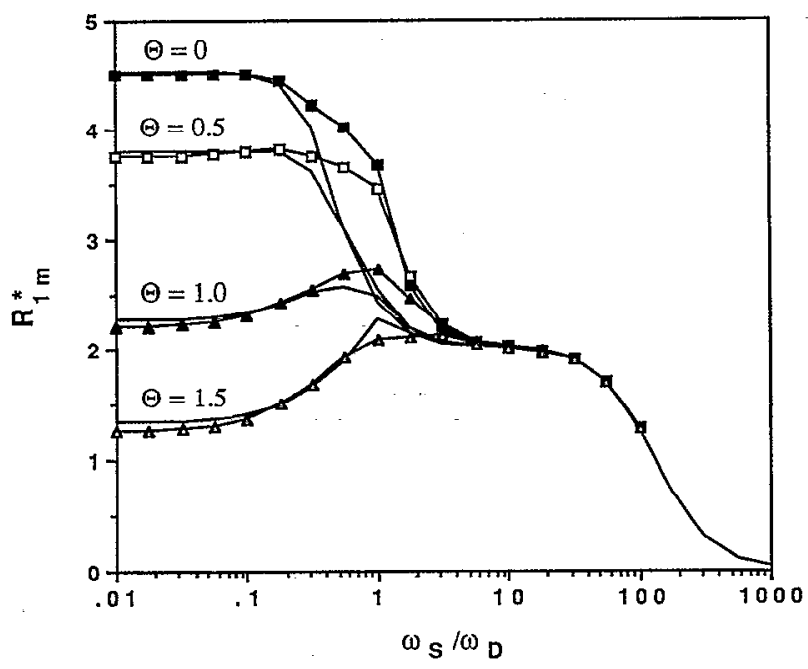

FIG. 1. Reduced dipolar relaxation rate $R_{1 m}^{*}$ against the reduced electron larmor frequency $\omega_{S} / \omega_{D}$ for $S=1$ (no symbols) and $S=2$ (symbols) with $\tau_{C} \omega_{D}=5$. $\Theta$ is the polar angle of the $I$ spin in the molecular coordinate system which diagonalizes the $z$ fs tensor.

about $\omega_{\Gamma} \tau_{c}=1$ and $\omega_{S} \tau_{c}=1$, respectively, with relative amplitudes of 3:7 [Eq. (3)]. Type-1 features result physically from the lifting of the degeneracy of the electron spin energy levels by the Zeeman interaction. These features occur only in the regime of field strengths where the Zeeman energy is larger than the zfs energy; they are suppressed in the region where $\omega_{S} / \omega_{D}<1$.

Type 2. These result from an alteration in the meansquared magnetic dipole coupling energy which occurs when the quantization axis of the precessional motion of the electron spin changes from the molecular coordinate system in the zfs limit to the external field axis in the Zeeman limit. Type-2 features are strongly $\Theta$ dependent, as illustrated in Fig. 1, which shows profiles for $S=1$ (no symbols) and $S=2$ (symbols). The midfield point of the type-2 feature lies in the intermediate regime $\omega_{S} / \omega_{D} \cong 1$, its precise position depending on the spin quantum number (see below).

Type 3. A local $R_{1 m}$ maximum may occur in the intermediate regime due to the near crossing or "pinching" of the electron spin energy levels. For $S=1$, this is a relatively small feature, the amplitude of which depends strongly on $\theta$.

Type 4. The appropriate definition of the molecular reorientational correlation time changes from $\tau_{R}^{(1)}$ in the zfs limit to $\tau_{R}^{(2)}$ in the Zeeman limit (see above). This change is associated physically with the change in quantization axis of the electron spin precessional motion in the intermediate regime. When $\tau_{R}$ contributes significantly to the dipolar correlation time, the type- 4 feature appears in the dispersion profile as a monotonic decrease in $R_{1 m}^{*}$, which may drop by as much as a factor of 3 when the field strength rises through the intermediate regime.

Type 5. A local maximum in $R_{1 m}^{*}$ may occur at high field due to magnetic field dependence in the electron spin relaxation time, which, in the Zeeman limit, is given by ${ }^{4}$ 


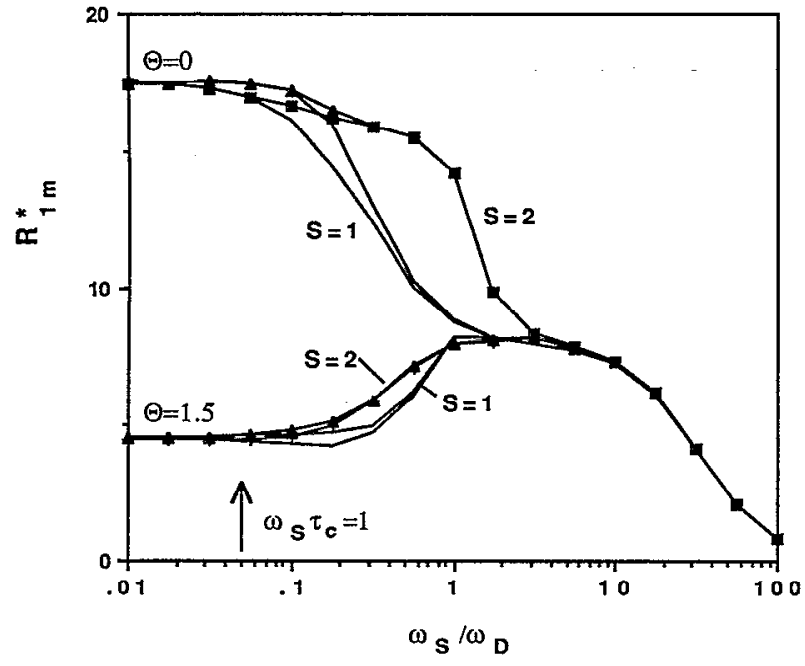

FIG. 2. Reduced dipolar relaxation rate $R_{1 m}^{*}$ against the reduced electron larmor frequency $\omega_{S} / \omega_{D}$ for $S=1$ and $S=2$ with $\tau_{c} \omega_{D}=5$. Curves calculated for the zfs limit are labeled by the value of $\Theta$. For each value of $S$ and $\Theta$ two curves are shown, the upper corresponding to the total $R_{1 m}^{*}$ (as is shown in Fig. 1), the lower corresponding to the low frequency part of $R_{1 m}^{*}$ [the term proportional to $j\left(\omega_{1}\right)$ in Eq. (1)].

$$
\tau_{S}^{-1}=\frac{1}{\tau_{S}^{(0)}}\left(\frac{0.2}{1+\omega_{S}^{2} \tau_{v}^{2}}+\frac{0.8}{1+4 \omega_{S}^{2} \tau_{v}^{2}}\right)
$$

$\tau_{S}^{(0)}$ is the low field limiting value of $\tau_{S}$, and $\tau_{v}$ is the correlation time for the motions which produce electron spin relaxation. The field dependence of $\tau_{S}$ described by Eq. (10) is suppressed in the region $\mathscr{H}_{\text {zfs }}>\mathscr{H}_{Z}$, where the level splittings are determined principally by the zfs interaction and are largely field independent. Thus the type-5 feature is a characteristic feature of the vicinity of the Zeeman limit and is suppressed where $\omega_{S} / \omega_{D}<1$.

\section{RESULTS}

Reduced field dispersion profiles of the $T_{1}$ NMR-PRE for spin systems with $S=1,3 / 2,2$, and $5 / 2$ have been calculated using the program PARELAX. The results are compared and summarized in Figs. 1-9. In these plots, the intermediate regime between the zfs and Zeeman limits occurs roughly in the vicinity of $\omega_{S} / \omega_{D}=1$, the position depending on the spin quantum number $S$ (see below). In the zfs limit and in the intermediate regime, but not in the Zeeman limit, $R_{1 m}^{*}$ depends on the position of the $I$ spin in the molecular coordinate system. Calculations were performed only for the range of correlation times $\omega_{D} \tau_{c}>1$. In the opposite situation $\left(\omega_{D} \tau_{c}<1\right)$, effects of the zfs interaction are effectively averaged to zero and in the limit $\omega_{D} \tau_{c} \ll 1$ have no influence on $R_{1 m}$. In this situation, Zeeman-limit theory is valid, even at low field strengths where $\omega_{S} / \omega_{D} \ll 1$ (see Ref. 8). Uniaxial molecular symmetry was assumed, i.e., that the zfs parameter $E=0$. Thus specification of the molecular geometry requires specification of only the polar angle $\Theta$ between the $I-S$ vector and the principal axis $\hat{z}$ of the zfs tensor.

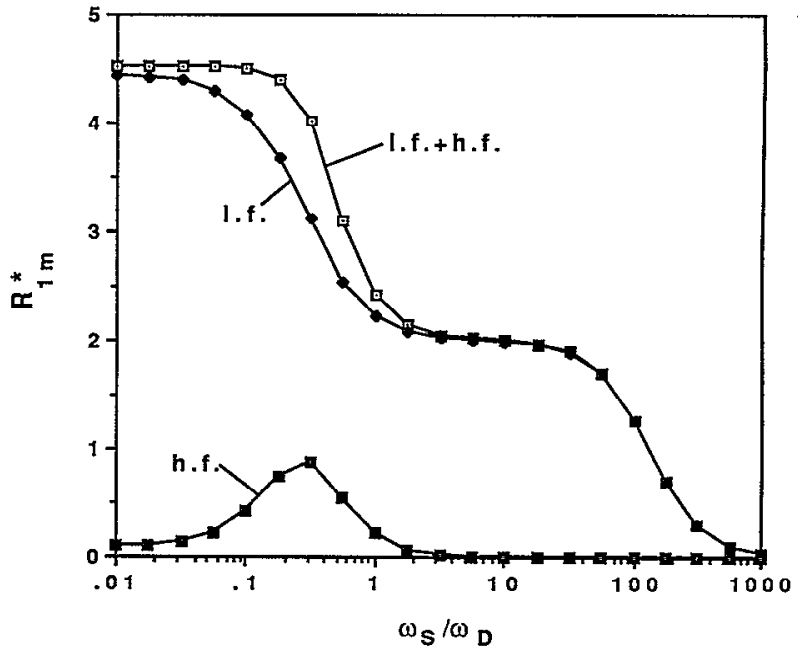

FIG. 3. Rcduced dipolar relaxation rate $R_{1 m}^{*}$ against the reduced electron larmor frequency $\omega_{S} / \omega_{D}$ for $S=1, \Theta=0$, and $\tau_{c} \omega_{D}=5$ The total paramagnetic relaxation rate is decomposed into low frequency terms [those proportional to $\left.j\left(\omega_{I}\right)\right]$ and high frequency terms [proportional to $j\left(\omega_{\mu}\right.$ $\left.\left.+\omega_{v} \pm \omega_{l}\right)\right]$

\section{A. Field dispersion profiles for integer spin}

Figure 1 shows field dispersion profiles for $S=1$ (no symbols) and $S=2$ (symbols) for selected values of $\Theta$ with $\omega_{D} \tau_{c}=5$. When plotted in reduced form, the profiles for $S=1$ and $S=2$ are similar with respect to their qualitative features. The reduced profiles for all $\Theta$ and $S$ values coalesce in the Zeeman limit $\left(\omega_{S} / \omega_{D} \gg 1\right)$, as required by Eq. (3), which is independent of molecular geometry and, in reduced form, of $S$. In the zfs limit, the reduced profiles depend strongly on $\Theta$, but only very weakly on $S$. The principal qualitative features of the profiles are a high-field Zeeman-type dispersion (type 1) that is centered at $\omega_{1} \tau_{c}=1$ (which occurs at $\omega_{S} / \omega_{D}=132$ in Fig. 1), and a

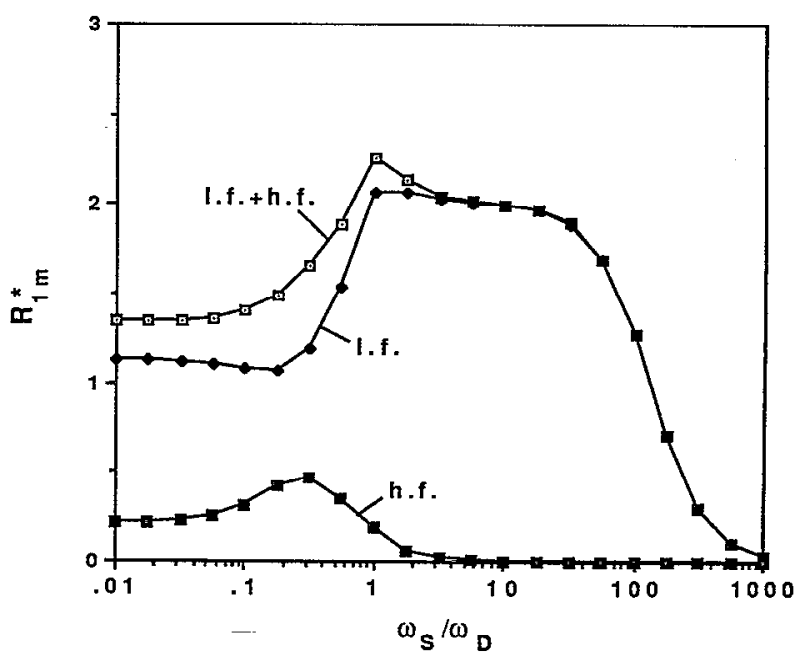

FIG. 4. A plot of the reduced field dispersion profile for $S=1$, as in Fig. 3 , except with $\theta=1.5$. 


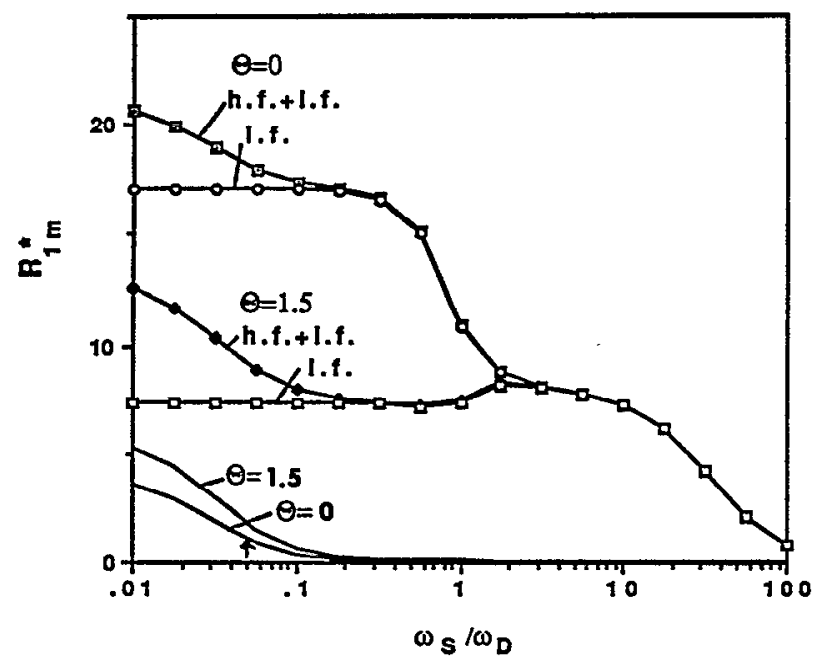

FIG. 5. A plot of the reduced field dispersion profile for $S=3 / 2 ; \theta=0$ and $\Theta=1.5$; and $\tau_{c} \omega_{D}=20$ The total relaxation rate (1.f. + h.f.) is decomposed into low frequency (l.f.) and high frequency (h.f.) terms. The h.f. terms are shown separately at the bottom.

requantization type-2 feature that is centered near $\omega_{S} / \omega_{D}$ $=1$. Effects of a type-3 feature also contribute in a minor way near $\omega_{S} / \omega_{D} \cong 1$. However, the type-4 feature is entirely absent since $\omega_{D} \tau_{c}$ was taken to be constant, which is equivalent to assuming that $\tau_{R} \gg \tau_{c}$, i.e., that $\tau_{R}$ does not contribute to $\tau_{c}$. Also, no low-field type-1 feature (the Zeeman-type dispersion that is centered about $\omega_{S} \tau_{c}=1$ ) is present in the profiles, since this feature is suppressed when it occurs in the vicinity of the zfs limit (where $\mathscr{H}_{Z}$ $<\mathscr{H}_{\text {zfs }}$ ).

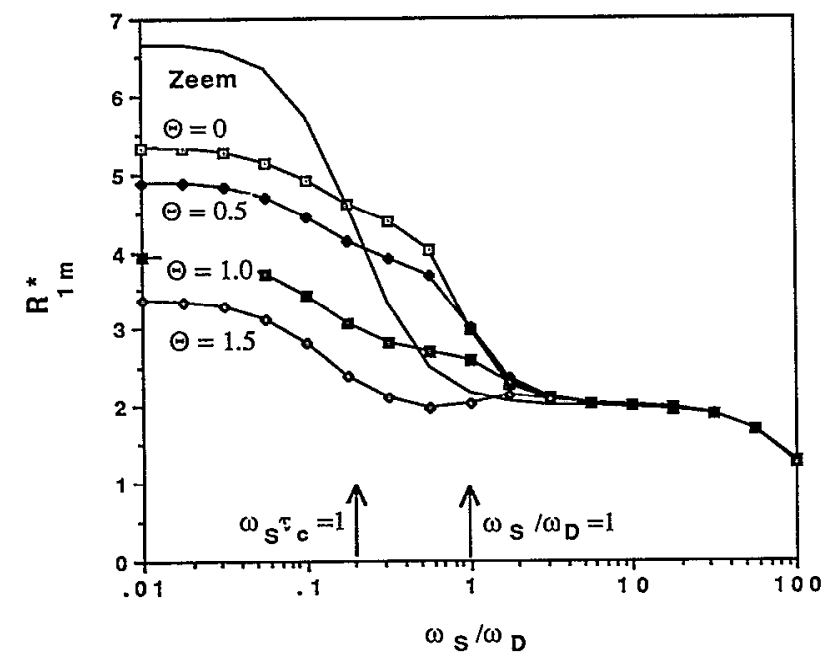

FIG. 6. The reduced field dispersion profile of $R_{\mathrm{l} m}^{*}$ for $S=3 / 2$ with $\tau_{c} \omega_{D}=5$. Curves from top to bottom correspond to $\Theta=0,0.5,1.0$, and 1.5 , where $\theta$ is the polar angle of the $I$ spin in the molccular coordinate system. The type- 6 feature lies to the low field of $\omega_{s} \tau_{c}=1$. The Zeemanlimit (Zeem) curve is shown for comparison. The type-6 features are centered to the low field of $\omega_{S} \tau_{c}=1$.

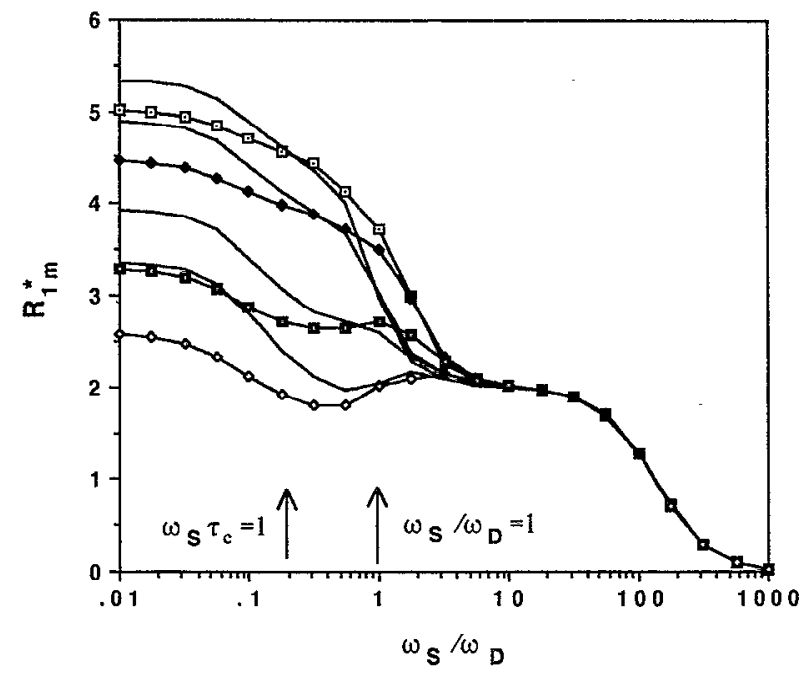

FIG. 7. The reduced field dispersion profile of $R_{1 m}^{*}$ for $S=3 / 2$ (no symbols) and $S=5 / 2$ (symbols) with $\tau_{c} \omega_{D}=5$. Curves from top to bottom correspond to $\Theta=0,0.5,1.0$, and 1.5 , where $\Theta$ is the polar angle of the $I$ spin in the molecular coordinate system.

In the calculations of Fig. 1, the low-field type- 1 dispersion that is predicted by Zeeman-limit theory is centered about $\omega_{S} / \omega_{D}=0.2$; clearly, its contribution to the $S=1$ and $S=2$ profiles is very minor if not negligible. Further investigating this point, Fig. 2 shows profiles for $S=1$ and $S=2$ calculated assuming $\omega_{D} \tau_{c}=20$. In Fig. 2, each $S$ and $\Theta$ value is associated with a pair of curves, the upper of which describes the total $R_{1 m}^{*}$ as is shown in Fig. 1, and the lower shows the low frequency (1.f.) portion of $R_{1 m}^{*}$ [i.e., the term proportional to $j\left(\omega_{I}\right)$ in Eq. (1)]. The total

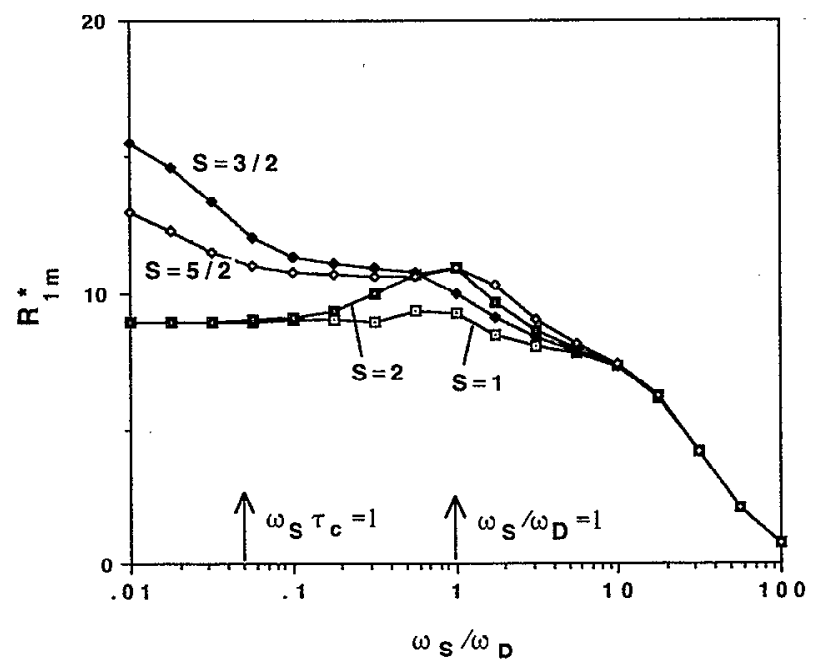

FIG. 8. Reduced field dispersion profiles of $R_{1 m}^{*}$ for $S=1,3 / 2,2$, and $5 / 2$ with $\Theta=0.955 \mathrm{rad}$ and $\tau_{c} \omega_{D}=20$. In this plot, the type- 4 feature is absent (since $\tau_{c}$ is constant) and the type-2 feature is minimized by the choice of $\Theta$. Thus the role of the type-3 feature, which occurs in the vicinity of $\omega_{S} / \omega_{D}=1$, is emphasized. 


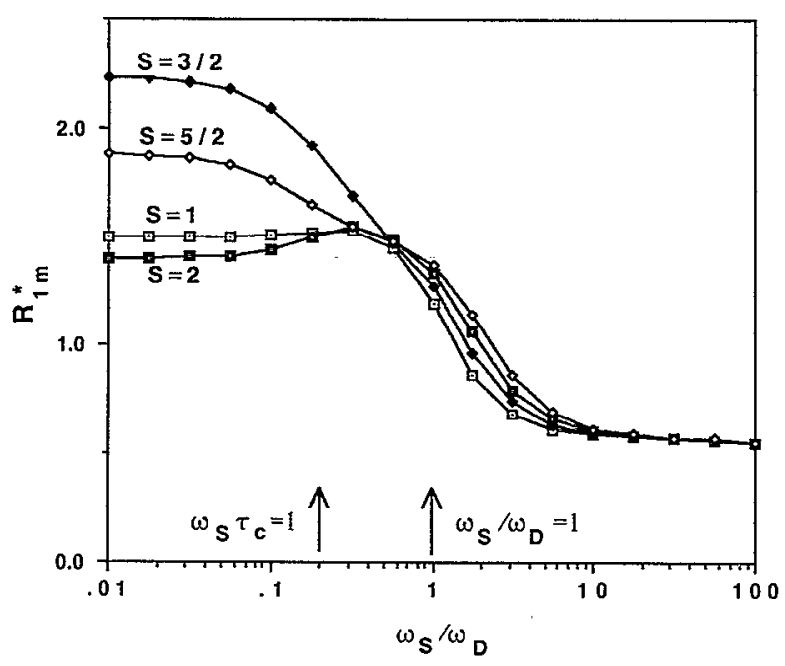

FIG. 9. Field dispersion profiles for $S-1,3 / 2,2$, and $5 / 2$ calculated with $\tau_{S} \omega_{D}=5$ (independent of field strength), $\tau_{R}^{(2)} \omega_{D}=5$, and $\Theta=0.955$ (this $\Theta$ value minimizes type- 2 features). The pronounced decrease in $R_{1 m}^{*}$ that occurs near $\omega_{S} / \omega_{D} \approx 1.5$ results predominantly from type-4 features (i.e., due to the redefinition of the reorientational correlation time that is associated with requantization of the electron spin).

$R_{I m}^{*}$ will be discussed first; the relative high frequency and low frequency terms in $R_{1 m}^{*}$ are discussed below.

A comparison of Figs. 1 and 2 shows that when $\omega_{D} \tau_{c}$ is lengthened to 20 , the Zeeman-type dispersions are displaced to lower values of $\omega_{S} / \omega_{D}$, their positions indicated by arrows in Fig. 2 . The high-field, type-1 feature remains, its midfield value shifted to $\omega_{S} / \omega_{D}=33$, while the lowfield, type-1 feature is almost entirely suppressed.

Close inspection of Figs. 1 and 2 also shows the interesting fact that the effects of the zfs on the shape of the dispersion profile are largely independent of $\tau_{c}$. Variations in $\tau_{c}$ are reffected in the scaling of the profile, as well as in the midfield position of the high-field, type- 1 feature, which occurs at $\omega_{I} \tau_{c}$. However, when $\omega_{D} \tau_{c}>1$, the profiles for different $\omega_{D} \tau_{c}$ are quite similar throughout the range of field strengths where $\mathscr{H}_{\text {zfs }} \gtrsim \mathscr{H}_{Z}$ (or equivalently, where $\omega_{S} / \omega_{D} \lesssim 1$ ). In fact, for integer spin systems only, it would be useful to further normalize the profile with respect to $\omega_{D} \tau_{c}$ by plotting $R_{1 m}^{*}\left(\omega_{\nu} \tau_{c}\right)^{-1}$ vs $\omega_{D} / \omega_{S}$, in which case, the profiles for different integer spin values in the region $\omega_{S} / \omega_{D} \lesssim 1$ would be nearly the same, largely independent of $\tau_{c}$. However, this form is less useful for half-integer spins (see below) and is not used here.

The principal difference in the profiles for $S=1$ and $S=2$ is that the requantization features (types 2 and 3 ) for $S=2$ are diplaced along the $\omega_{S} / \omega_{D}$ axis relative to the corresponding features for $S=1$. This displacement is $\Theta$ dependent, being in the up-field direction by a factor $>2$ at $\Theta=0$ and in the down-field direction by a much smaller factor for $\Theta=\pi / 2$. The $S$ dependence and a $\Theta$ dependence of the midfield position of the type-2 features is quite unexpected. For $S=1$, the midfield point of the $\Theta=0$ profile is to the low field of the midfield point of the $\theta=\pi / 2$ profile. For $S=2$, the $\Theta$-dependence is in the opposite di- rection, $\Theta=0$ occuring to high field of $\Theta=\pi / 2$. At present, we can offer no simple physical rationale for this effect.

\section{B. Role of the high and low frequency terms}

To better understand the profiles, the high and low frequency parts of $R_{1 m}^{*}$ were calculated and plotted separately. For $\omega_{D} \tau_{c} \gg 1$, it was found that the profiles reflect almost entirely the low frequency term of $R_{1 m}^{*}$ [that proportional to $j\left(\omega_{I}\right)$ ]. This is illustrated in Fig. 2, where the l.f. contribution is plotted along with the total $R_{1 m}^{*}$ (h.f. +1.f.). The h.f. terms are negligible in the zfs and Zeeman limits and become significant, though still very small, only in the regime near $\omega_{S} / \omega_{D}=0.1$. However, the h.f. term becomes relatively more important as $\omega_{D} \tau_{c}$ decreases toward unity. Figures 3 and 4 illustrate the situation for $S=1, \omega_{D} \tau_{c}=5$. The h.f. terms vanish in the Zeeman limit, but not in the zfs limit, where they are greater for equatorial positions $[\Theta=\pi / 2$ (Fig. 4)] than for axial positions $[\Theta=0$ (Fig. 3)]. This behavior conforms to the $\Theta$ dependence of Eq. (1). In the intermediate regime, the h.f. terms pass through a maximum and can be significant for all $\Theta$ values. It is the h.f. term that is responsible for type-3 features (i.e., local maxima in the dispersion profile near $\left.\omega_{S} / \omega_{D}=1\right)$, an example of which is present in the profile of Fig. 4.

\section{Dispersion profiles for half-integer spins}

Dispersion profiles for $S=3 / 2$, calculated with $\omega_{D} \tau_{c}$ $=20$ and $\Theta=0$ and 1.5, are shown in Fig. 5. These profiles differ qualitatively from those of Fig. 2 in that a substantial new feature is present in the low-field region. This feature results entirely from the high frequency part of $R_{1 m}^{*}$, specifically from the term containing $j\left(\omega_{-1 / 2}\right)$ in Eq. (4b), which represents the contribution of the $\left(m_{S}=+1 / 2 \leftrightarrow m_{S}\right.$ $=-1 / 2$ ) Kramers doublet. The h.f. terms of $R_{1 m}^{*}$ arise from single quantum transitions within the electron spin manifold. For all integer spins, these transitions occur, in zero field, at integral multiples of $\omega_{D}$ [e.g., Eqs. (4a) and (4c)]. Thus for integer spin in zero field, the h.f. terms are proportional to $j\left(n \omega_{D}\right)$. When $\omega_{D} \tau_{c}<1$, these terms are small compared to the 1.f. term. For half-integer spins, the $m_{S}= \pm 1 / 2, \pm 3 / 2, \pm 5 / 2, \ldots$ Kramers doublets are strictly degenerate in zero field. The $m_{S}= \pm 1 / 2 \mathrm{Kramers}$ doublet defines a single quantum transition which contributes a relatively low frequency term $j\left(\omega_{-1 / 2,1 / 2}\right)$ to the h.f. part of $R_{1 m}^{*}$ [Eqs. (4b) and (4d)]. This contribution, at low field, can be comparable in magnitude to the 1.f. term. The $m_{S}= \pm 1 / 2$ splitting $\omega_{-1 / 2,1 / 2}$ depends on the relative orientations of the molecular and laboratory coordinate axes and lies in the range $0<\omega_{-1 / 2,1 / 2}<\omega_{S}$. Thus this feature is broader than, and centered somewhat to the low field of the Zeeman type-1 feature at $\omega_{S} \tau_{c}=1$.

The feature due to the $m_{S}= \pm 1 / 2$ Kramers doublet represents a sixth type, which is characteristic of halfinteger electron spins in the low-field region. While it is similar in physical origin to the low-field, type-1 feature, its properties differ considerably. The amplitude of the type- 6 dispersion is $\Theta$ and $S$ dependent and is considerably 
smaller than for type 1 , since only one pair of spin levels contributes. The feature is centered somewhat to the low field of $\omega_{S} \tau_{c}=1$, compared to the type-1 feature, which is centered precisely at $\omega_{S} \tau_{c}=1$.

It was pointed out above that for integer spins, the qualitative character of the profile in the zfs-dominated region has little dependence on $\omega_{D} \tau_{c}$ (aside from scaling the entire profile); this is particularly true when $\omega_{D} \tau_{c} \gg 0$. It is not true, however, for half-integer spins, since the position of the type- 6 feature along the $\omega_{S} / \omega_{D}$ axis varies as $\tau_{c}^{-1}$. The dependence of the $S=3 / 2$ profile shape on the reduced correlation time $\omega_{D} \tau_{c}$ is illustrated in Figs. 5 and 6 , which were calculated for $\omega_{D} \tau_{c}=20$ and $\omega_{D} \tau_{c}=5$, respectively. Although there is some overlap of the features in Fig. 6, the underlying structure of the profiles is evident, consisting of a type- 1 feature at $\omega_{r} \tau_{c}=1$, type- 2 and type- 3 features near $\omega_{S} / \omega_{D}=1$, and a type-6 feature to low field of $\omega_{s} \tau_{c}=1$. Type 4 and 5 features are absent since $\tau_{c}$ was assumed to be constant. For comparison, the low-field type-1 feature of Zeeman-limit theory is shown in Fig. 6.

Figure 7 compares dispersion profiles for $S=3 / 2$ (no symbols) and $S=5 / 2$ (symbols) for $\omega_{D} \tau_{c}=5$. The profiles for given $\Theta$, but different $S$ are clearly similar in structure, showing a type-1 feature centered where $\omega_{\Gamma} \tau_{c}=1$, type-2 and 3 features in the intermediate regime where $\omega_{S} / \omega_{D} \approx 1$, and a type- 6 feature near, but somewhat to the low field of $\omega_{S} \tau_{c}=1$. The type- 2 and 3 features exhibit shifts in midfield position that are similar to those found for $S=1$ and $S=2$; namely, these features are shifted to high field for $S=5 / 2$ relative to $S=3 / 2$ when $\theta \approx 0$, and in the opposite direction when $\Theta \approx 1.5$. Also, the type- 6 features are smaller in amplitude for $S=5 / 2$ than for $S=3 / 2$, since for $S=5 / 2$, the $m_{S}= \pm 1 / 2$ Kramers doublet accounts for a smaller fraction of the total spin magnetization.

\section{Type-3 and type-4 features}

Figure 8 compares the calculated dispersion profiles at $\Theta=0.9553$, where $P_{2}(\cos \Theta)=0$. At this $\Theta$ value, the magnetic dipole coupling energy is the same as the value averaged over the surface of a sphere and thus there is no change in mean-squared dipole coupling energy upon passing from the $\mathrm{zfs}$ to the Zeeman limit. This choice of $\Theta$ minimizes the type- 2 feature. In addition, the type- 4 feature is strictly absent in these profiles since $\tau_{c}$ was taken to be field independent (equivalently, $\tau_{R}$ is taken to be very long). Thus, the profiles of Fig. 8 emphasize the role of the type-3 feature. Interestingly, it was found that this feature, which is present though small for all $S$ values, is considerably larger for $S=2$ than for the other values of $S$.

And finally, the role of the type- 4 feature (that due to redefinition of $\tau_{R}$ in the intermediate regime) was examined (Fig. 9). In these calculations, values of $\omega_{D} \tau_{R}^{(2)}=5$ and $\omega_{D} \tau_{S}=5$ were assumed and $\Theta$ was set equal to 0.955 to minimize the type- 2 feature. The type- 4 feature consists of a decrease in $R_{1 m}^{*}$ in the intermediate regime as $\tau_{R}$ drops from the zfs-limit value of $\tau_{R}^{(1)}$ to the Zeeman-limit value of $\tau_{R}^{(2)}$. This drop is apparent in Fig. 9 and is quantitatively quite similar for all $S$ values, integer and half-integer. It

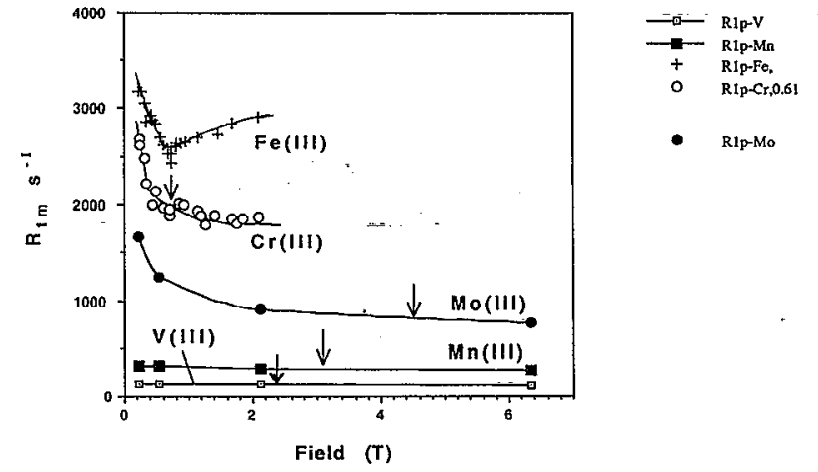

FIG. 10. Magnetic field dependence of $R_{1 \mathrm{~m}}$ of the methyl protons of tris-(acetylacetonato) $\mathrm{Me}$ (III) complexes in solution, where $\mathrm{Me}=\mathrm{V}$ (III), $\mathrm{Mn}$ (III), Mo(III), $\mathrm{Fe}$ (III), and $\mathrm{Cr}$ (III). Electronic spins $S$ and uniaxial zfs parameters $D$ (where known) of the complexes are $\mathrm{V}(\text { acac })_{3} S=1$, $|D| \cong 3 \mathrm{~cm}^{-1}$ (Ref. 24); $\mathrm{Mn}(a c a c)_{3} S=2, D=3.1 \mathrm{~cm}^{-1}$ (Ref. 30); $\operatorname{Mo}(a c a c)_{3}, \quad S=3 / 2 ; \quad \operatorname{Cr}(a c a c)_{3} \quad S=3 / 2, \quad D=0.6 \mathrm{~cm}^{-1}$ (Ref. 29); $\mathrm{Fe}(a c a c), S=5 / 2$. Arrows indicate the field value at which $\omega_{S} / \omega_{D}=1$.

should be recognized, however, that the results in the intermediate regime of Fig. 9 are only approximate since the generalized theory of Ref. 7 assumes that $\tau_{S} \ll \tau_{R}^{(2)}$. Even so, the curves of Fig. 9 are accurate in the limiting regimes, since Eqs. (1)-(3) do not depend on this assumption, and the behavior in the intermediate regime has at least semiquantitative validity.

\section{E. Summary}

In summary, it appears that for integer spin systems, all of the qualitative features of the dispersion profiles can be described satisfactorily in terms of the five types that have been described previously and are summarized in the Methods section above. For half-integer spins, a sixth type of feature, which contributes to the h.f. term of $R_{1 m}^{*}$ and arises specifically from the contribution of the $m_{S}= \pm 1 / 2$ Kramers doublet, is present. The type- 6 feature is centered somewhat to the low field of $\omega_{s} \tau_{c}=1$, which is the midfield position of the corresponding type-1 Zeeman feature. Its amplitude, which is $\Theta$ and $S$ dependent, is considerably smaller than the type-1 feature. Its amplitude, which is $\Theta$ and $S$ dependent, is considerably smaller than the type- 1 amplitude.

\section{COMPARISON WITH EXPERIMENT}

The tris-acetylacetonato complexes of the transition metal ions provide a homologous series that is useful for assessing the characteristic relaxation properties of different integer and half-integer spin values. These complexes, especially $\operatorname{Cr}(a c a c)_{3},{ }^{11,13,15}$ have been studied with respect to the influence of the zfs on the PRE. Field dependence of the $T_{1}$ relaxation has been reported for the methyl protons of the tris-acetylacetonato complexes of V(III) $(S=1)$; $\mathrm{Cr}$ (III) and $\operatorname{Mo}(\mathrm{III})(S=3 / 2) ; \operatorname{Mn}(\mathrm{III})(S=2)$; and $\mathrm{Fe}(\mathrm{III})(S=5 / 2)$. The available data ${ }^{11,15,22,23}$ at $298 \mathrm{~K}$ are summarized in Fig. 10. Arrows associated with various curves indicatc the ficld strength at which $\omega_{S}=\omega_{D}$, i.e., the 


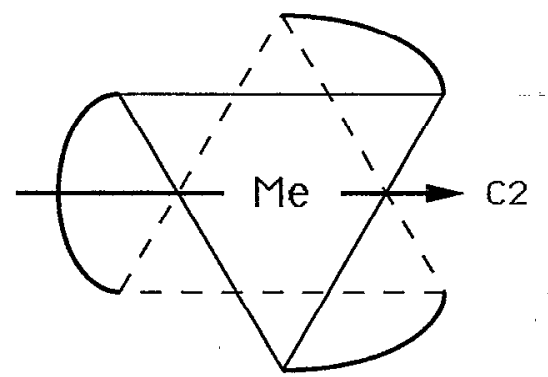

FIG. 11. A schematic view of $\mathrm{Me}(a c a c)_{3}$ complexes down the $D_{3}$ axis. A tetragonal Jahn-Teller distortion, as occurs in $\mathrm{Mn}(a c a c)_{3}$, reduces the $D_{3}$ point symmetry to $C_{S}$.

approximate field strength near which changes in electron spin quantization are expected to occur. No value of $D$ has been reported for $\mathrm{V}(a c a c)_{3}$, but values of several $\mathrm{cm}^{-1}$ are typical of $\mathrm{V}^{3+}{ }^{24}$

The striking qualitative observation in Fig. 10 is that for the integer spin systems, the profiles are very nearly field independent, while for the half-integer spin systems $R_{1 m}$ shows fairly strong field dependence at low field, increasing sharply as the field strength falls below $1 \mathrm{~T}$. This rise occurs to the low field of the cross-over point where $\omega_{S}=\omega_{D}$ and thus is the kind of qualitative behavior expected of a type- 6 feature. It is expected, in accord with experiment, that the integer spin systems will not exhibit low field features of this type.

The point group symmetry of the $\mathrm{V}(\text { acac })_{3}$, $\mathrm{Cr}(a c a c)_{3}, \mathrm{Mo}(a c a c)_{3}$, and $\mathrm{Fe}(a c a c)_{3}$ complexes is $D_{3}$ (Fig. 11). This differs from the situation for $\mathrm{Mn}(a c a c)_{3}$, in which the site symmetry of the metal ion is determined principally by a tetragonal Jahn-Teller distortion; the overall symmetry of the complex is $C_{S}$. The influence of $\mathrm{zfs}$ effects on the Mn(acac) 3 NMR-PRE profile has been discussed previously. ${ }^{7} \mathrm{~V}$ (III) [but not $\mathrm{Cr}$ (III), Mo(III), or $\mathrm{Fe}(\mathrm{III})]$ is likewise subject to Jahn-Teller distortion, which is generally along a three-fold coordination axis. Averaged values of $r$ and $\theta$ used in the theoretical simulations of the $R_{1 p}$ dispersion profiles were taken from X-ray crystal structure data for $\mathrm{Mn}(a c a c)_{3}$. The effective value of $r$ for the methyl protons was calculated as the mean value of $r^{-6}$ taken to the sixth power and then averaged over the $n=18$ methyl protons, i.c., $r=(1 / n) \Sigma\left(\left\langle r^{-6}\right\rangle\right)^{6}$, using structural data from two $x$-ray crystallographic studies. ${ }^{25,26}$ This calculation gave an averaged experimental value of $r=4.57 \AA$. Averaged $\Theta$ values, likewise extracted from the $x$-ray structural data, are $\theta_{\mathrm{av}}=1.03 \mathrm{rad}$ with respect to the fourfold coordination axis and $\theta_{\mathrm{av}}=0.94 \mathrm{rad}$ with respect to the three-fold axes. Scalar contributions to $T_{1}$ 's of $\mathrm{CH}_{3}$ protons were neglected, in accord with previous findings. ${ }^{27}$

Turning first to $\mathrm{V}(a c a c)_{3}(S=1)$, Fig. 12 shows the experimental data along with theoretical simulations based on Zeeman-limit theory (SBM) and the generalized theory (G.T.). Both fits assume the following parameters: $\tau_{S}^{(0)}=8$ ps; $g=1.7$ (Ref. 28 ),$r=4.7 \AA$, and $\tau_{v}=0.5 \mathrm{ps,} \mathrm{and} \mathrm{for} \mathrm{the}$ G.T. fit, $\Theta=0.94 \mathrm{rad}$ and $|D|=3.0 \mathrm{~cm}^{-1}$. SBM theory is

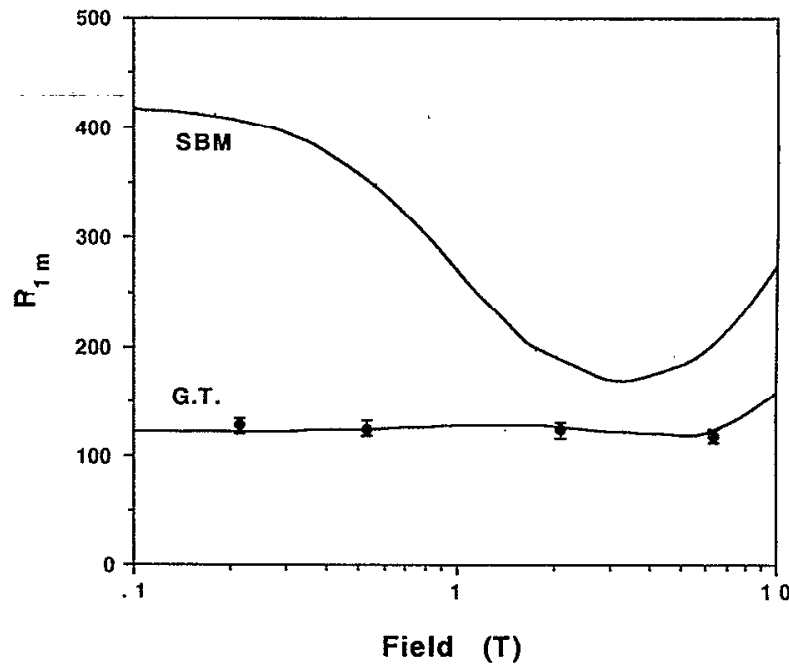

FIG. 12. Fits of Zeeman theory (SBM) and the generalized theory (G.T.) to experimental $R_{1 m}$ data (Ref. 11) for the methyl protons of $\mathrm{V}(a c a c)_{3}$. Theoretical fits used the following parameter values: $\tau_{S}=8 \mathrm{ps}$; $\tau_{R}^{(2)}=45 \mathrm{ps} ;|D|=3 \mathrm{~cm}^{-1} ; \Theta=0.94 \mathrm{rad} ; r=4.7 \AA ; \tau_{v}=0.5 \mathrm{ps}$.

incapable of satisfactorily simulating the data because of the large type-1 dispersive feature that occurs at $\omega_{S} \tau_{S}=1$ $(\sim 1 \mathrm{~T})$. Since $\omega_{D} \tau_{c}>1$, this feature is largely suppressed in the G.T. plot, which provides an excellent fit of the profile using realistic parameters. The nearly featureless character of the G.T. profile below $5 \mathrm{~T}$ results from several factors. A pronounced type-2 feature is absent, since the $\theta$ value for $\mathrm{V}(a c a c)_{3}, \theta_{\mathrm{av}}=0.94$, with respect to the threefold axis is very close to the value $\Theta_{\mathrm{av}}=0.955$ at which $P_{2}(\cos \theta)=0$. Similarly, the type-4 feature is nearly absent since $\tau_{S} \ll \tau_{R}^{(2)}$ and $\tau_{c} \cong \tau_{S}$. Also, the type-3 feature is very small since $\omega_{D} \tau_{c}$ is not much greater than 1 . The type $2-4$ features result in mild field dependence in the profile near $2-5 \mathrm{~T}$, but this is largely counteracted by contrary effects of the type- 5 feature. For these reasons, the profile is nearly flat below 7 T. The calculated G.T. profile does not depend in a sensitive way on $|D|$, but in the high field region ( $\left.B_{0}>5 \mathrm{~T}\right)$, both profiles depend rather sensitively on $\tau_{v}$.

The situation for $\operatorname{Mn}(a c a c)_{3}(S=2)$ has been discussed previously ${ }^{7}$ and is similar to that for $\mathrm{V}(a c a c)_{3}$. In particular, low-field Zeeman type- 1 and zfs type- 6 features are absent in the profile, as expected for integer spin.

For $\operatorname{Cr}(a c a c)_{3}(S=3 / 2)$, field dependence in the low field region of the profile $\left(\omega_{S} / \omega_{D}<1\right)$ due to a type 6 feature is expected, and field dependence qualitatively consistent with this behavior is present in the profile (Fig. 10). However, the application of the generalized theory to $\mathrm{Cr}(a c a c)_{3}$ is not appropriate, since $\tau_{S}$ in this case is quite long, $>870$ ps as measured from solution-phase electron spin resonance (ESR) linewidth data, ${ }^{29}$ and an assumption of the generalized theory, namely, that $\tau_{R}^{(2)} \ll \tau_{S}$ is not satisfied. The situation for $\mathrm{Fe}(a c a c)_{3}$ is similar.

For $\mathrm{Mo}(a c a c)_{3}, \tau_{S}$ is of the order of 30 ps (Ref. 11) and $\tau_{R}^{(2)}=45$ ps (Ref. 27), so that $\tau_{S}<\tau_{R}^{(2)}$ and thus the generalized theory should at least be approximately valid. For $\mathrm{Mo}^{3+},|D|$ is typically of the order of 1 to a few $\mathrm{cm}^{-1}$. 


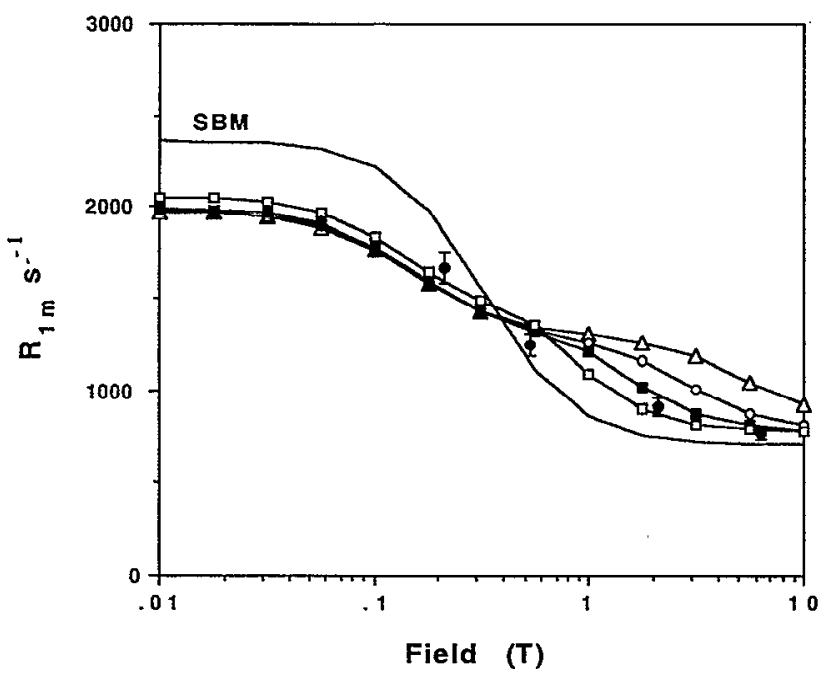

FIG. 13. Field dispersion profiles for Mo(acac) $)_{3}$. Experimental data, taken from Ref. 11, are shown as solid circles with error bars. The five curves are theoretically calculated field dispersion profiles based on Zeeman-limit theory (SBM) and on the generalized theory of Ref. 7 (curves with symbols). The calculations assume $r=4.5 \AA, \tau_{R}^{(2)}=44 \mathrm{ps,}$ and $g=2.2$. In addition, the Zeeman-limit calculations assume $\tau_{S}=25 \mathrm{ps,}$ while calculations using the generalized theory assume that $\tau_{S}=22.5 \mathrm{ps}$, $\Theta=1.02 \mathrm{rad}$, and, for the four curves, $D=4.5(\Delta) ; 2(O) ; 1(\square)$; and 0.5 $\mathrm{cm}^{-1}(\square)$.

Thus $\omega_{D} \tau_{c}>1$ and the zfs should influence the functional form of the profile. Experimental $R_{1 m}$ data for $\mathrm{Mo}(a c a c)_{3}$ are shown in Fig. 13 as solid circles with error bars. The magnetic field dependence of the data is significantly milder than is predicted by Zeeman-limit (SBM) theory, suggesting the importance of zfs effects. Also shown in the figure are curves calculated assuming four values of $|D|$ $\left(|D|=4.5,2,1\right.$, and $\left.0.5 \mathrm{~cm}^{-1}\right)$. Values of $r, \tau_{R}^{(2)}$, and $\Theta$ were fixed as described above, and $g$ was taken to be 2.25 . Aside from the scaling factor, which is strongly determined by $\tau_{S}$, the shape of the profile is determined principally by $|D|$, the best fit occurring for $|D|=0.5-1 \mathrm{~cm}^{-1}$. The drop in the calculated G.T. profile near $\sim 1-4 \mathrm{~T}$ is due primarily to a type-4 feature, and the drop near $0.1 \mathrm{~T}$ is due to a type- 6 feature. The SBM profile contains only a type-1 dispersive feature centcred near $0.3 \mathrm{~T}$. Static magnetic measurements of the $D$ parameter have not been reported (but see Ref. 11, in which a somewhat larger value $D=4.5$ $\mathrm{cm}^{-1}$ is suggested), and the solution phase ESR spectrum is unobservable due to the very short $\tau_{S}$. A fuller determination of the NMR-PRE profile would be particularly useful in this case, optimally at a lower temperature where $\tau_{S}$ is shorter and $\tau_{R}^{(2)}$ longer, as well as at lower field values.

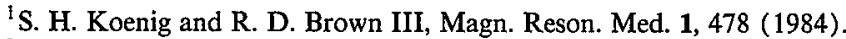

${ }^{2}$ I. Solomon, Phys. Rev. 99, 559 (1955).

${ }^{3}$ N. Bloembergen, J. Chem. Phys. 27, 572 (1957); 27, 595 (1957).

${ }^{4}$ N. Bloembergen and L. O. Morgan, J. Chem. Phys. 34, 842 (1961).

${ }^{5}$ R. R. Sharp J. Chem. Phys. 93, 6921 (1990).

${ }^{6}$ T. Bayburt and R. R. Sharp, J. Chem. Phys. 92, 5892 (1990).

${ }^{7}$ R. R. Sharp, J. Magn. Reson. 100, 491 (1992).

${ }^{8}$ R. R. Sharp, J. Chem. Phys. (to be published).

${ }^{9}$ U. Lindner, Ann. Phys. (Leipzig) 16, 319 (1965).

${ }^{10}$ D. T. Pegg and D. M. Doddrell, Aust. J. Chem. 31, 475 (1978).

${ }^{11}$ D. T. Pegg, D. M. Doddrell, M. R. Bendall, and A. K. Gregson, Aust. J. Chem. 29, 1885 (1976).

${ }^{12}$ H. L. Friedman, M. Holz, and H. G. Hertz, J. Chem. Phys. 70, 3369 (1979).

${ }^{13}$ T. R. Chen, S-J. Den, and L.-P. Hwang, Proc. Natl. Sci. Council (Republic of China) (A) 8, 224 (1984).

${ }^{14}$ L.-P. Hwang and C.-Y. Ju, J. Chem. Phys. 83, 3775 (1985).

${ }^{15}$ P.-L. Wang, J.-H. Lee, S.-M. Huang, and L.-P. Hwang, J. Magn. Reson. 73, 277 (1987).

${ }^{16}$ N. Benetis, J. Kowalewski, L. Nordenskiold, H. Wennerstrom, and P.-O. Westlund, Mol. Phys. 48, 329 (1983).

${ }^{17}$ P.-O. Westlund, H. Wennerstrom, L. Nordenskiold, J. Kowalewski, and N. Benetis, J. Magn. Resoll. 59, 91 (1984).

${ }^{18}$ N. Benetis and J. Kowaleski, J. Magn. Reson. 65, 13 (1985).

${ }^{19}$ L. Banci, I. Bertini, F. Briganti, and C. Luchinat, J. Magn. Reson. 66, 58 (1986).

${ }^{20}$ S. Szymanski, A. M. Gryff-Keller, and G. Binsch, J. Magn. Reson. 68, 399 (1986).

${ }^{21}$ W. T. Huntress, Adv. Nucl. Magn. Reson. Spectrosc. 4, 2 (1970).

${ }^{22}$ V. M. Nekipelov and A. N. Kitaigorodskii, J. Struct. Chem. 20, 181 (1979).

${ }^{23}$ A. N. Kitaigorodskii, V. M. Nekipelov, and K. I. Zamaraev, Dokl. Akad. Nauk. SSSR 235, 622 (1977).

${ }^{24} \mathrm{~A}$ good discussion of $\mathrm{V}^{3+}$ has been given by $\mathrm{A}$. Carrington and $\mathrm{A} . \mathrm{D}$ McLachlan, Introduction to Magnetic Resonance (Harper and Row, New York, 1967), pp. 158-163.

${ }^{25}$ J. P. Fackler, Jr. and A. Avdeef, Inorg. Chem. 13, 1864 (1974).

${ }^{26}$ B. R. Stults, R. S. Marianelli, and V. W. Day, Inorg. Chem. 18, 1853 (1979).

${ }^{27}$ D. M. Doddrell, D. T. Pegg, M. R. Bendall, and A. K. Gregson, Chem. Phys. Lett. 40, 142 (1976).

${ }^{28}$ This is typical of $g_{1}$ values given by A. Abragam and B. Bleaney, Electron Paramagnetic Resonance of Transition Ions (Clarendon, Oxford, 1970), p. 429.

${ }^{29}$ G. P. Vishnevskaya and B. M. Kozyrev, Dokl. Akad. Nauk. SSSR 185, 1072 (1968).

${ }^{30}$ A. K. Greason, D. M. Doddrell, and P. Healy, Inorg. Chem. 17, 1216 (1978). 